\title{
No Correlation between Serum Progranulin Levels and Severity of Diabetic Retinopathy in Chinese People with Type 2 Diabetes and Normal or Mildly Impaired Renal Function
}

haiyan cheng

Wuxi People's Hospital https://orcid.org/0000-0001-5085-095X

lan $\mathrm{xu}$

Wuxi People's Hospital

ruifang bu

Wuxi People's Hospital

cheng jiang

Wuxi People's Hospital

lijun yao

Wuxi People's Hospital

wenjun wu ( $D$ wuwenjung@163.com )

https://orcid.org/0000-0001-9746-8694

Research article

Keywords: Diabetes Mellitus, Progranulins, Diabetic Retinopathy, Renal Insufficiency

Posted Date: February 3rd, 2020

DOI: https://doi.org/10.21203/rs.2.22522/v1

License: (c) (i) This work is licensed under a Creative Commons Attribution 4.0 International License.

Read Full License 
No Correlation between Serum Progranulin Levels and Severity of Diabetic

Retinopathy in Chinese People with Type 2 Diabetes and Normal or Mildly Impaired Renal Function

Hai-Yan Cheng ${ }^{\mathrm{a} \#}$, Lan Xü ${ }^{\mathrm{a} \#}$, Rui-Fang Bu ${ }^{\mathrm{a}}$, Cheng Jiang ${ }^{\mathrm{b}}$, Li-Jun Yao ${ }^{\mathrm{a}, \mathrm{c}}$, Wen-Jun $\mathrm{Wu}^{\mathrm{a}^{*}}$

aDepartment of Endocrinology, the Affiliated Wuxi People's Hospital of Nanjing Medical University, Wuxi, China

'Department of Laboratory, the Affiliated Wuxi People's Hospital of Nanjing Medical University, Wuxi, China

${ }^{\mathrm{c}}$ Department of Endocrinology, Xuzhou Cancer Hospital, Xuzhou, China

*Correspondence author:

Wen-Jun Wu, Department of Endocrinology, the Affiliated Wuxi People's Hospital of Nanjing Medical University, No. 299, Qingyang Road, Wuxi City, Jiangsu Prov. China 214023; Phone: +86 15358008856; Email: wuwenjung@163.com. \#Co-first authors.

\begin{abstract}
Background: Progranulin (PGRN), a novel pro-inflammatory adipokine, was reported to be related to the development and progression of diabetic retinopathy(DR). However, recently PGRN was established as a renal function-dependent protein, but
\end{abstract}


no data regarding PGRN and DR excluded the influence of decreased renal elimination. This study aimed to investigate the correlation between serum PGRN and DR excluding the effect of deteriorating renal function.

Methods: 338 subjects with estimated glomerular filtration rate (eGFR) $\geq 60$ $\mathrm{ml} / \mathrm{min} / 1.73 \mathrm{~m}^{2}$ were divided into four groups of normal controls $(\mathrm{NC})(\mathrm{n}=76)$, simple diabetes mellitus $(\mathrm{SDM})(\mathrm{n}=195)$, nonproliferative diabetic retinopathy $(\mathrm{NPDR})(\mathrm{n}=41)$, and proliferative diabetic retinopathy $(\mathrm{PDR})(\mathrm{n}=26)$. Serum PGRN was quantified by enzyme-linked immunosorbent assay, and further analyses of serum PGRN in different groups were conducted.

Results: There was no significant difference of serum PGRN between NC, SDM, NPDR, and PDR groups $(P>0.05)$. Serum PGRN in all subjects negatively and significantly correlated with eGFR $(r=-0.144, P<0.05)$, triglycerides and glucose (TyG) index $(r=-0.127, P<0.05)$, and triglyceride (TG) $(r=-0.132, P<0.05)$, while positively and significantly correlated with low-density lipoprotein cholesterol (LDL-C) $(r=0.140, P<0.05)$. Multivariate stepwise regression analysis indicated only LDL-C $(\beta=1.030, P<0.05)$ was independently associated with serum PGRN.

Conclusions: We demonstrated that serum PGRN levels did not correlate with severity of DR in Chinese patients with type 2 diabetes and eGFR $\geq 60 \mathrm{ml} / \mathrm{min} / 1.73 \mathrm{~m}^{2}$, but positively and independently correlated with LDL-C. 
Key words: Diabetes Mellitus; Progranulins; Diabetic Retinopathy;

Renal Insufficiency

\section{Background}

Diabetic retinopathy (DR), the most common microvascular complication of type 2 diabetes, is a leading cause of vision impairment and blindness in working-age population globally[1,2]. Among diabetic populations, the estimated prevalence of DR is $34.6 \%$, which brings a substantial worldwide public health burden[1].In the last decade, the clinical management of DR have been dramatically changed by the using 
of anti-vascular endothelial growth factor (VEGF) drugs and corticosteroids[3,4]. However, because of the limited efficacy and potential adverse effects, a comprehensive understanding of the pathophysiology of DR is urgently needed for the development of therapy.

Recently, increasing evidence points to chronic low-grade inflammation as a crucial contributor to the development and progression of DR[5]. Various pro-inflammatory cytokines and chemokines, such as interleukin-6 (IL-6), tumor necrosis factor- $\alpha$ (TNF- $\alpha$ ), and monocyte chemoattractant protein-1 (MCP-1), are elevated in serum and ocular samples (vitreous and aqueous humor) from patients with DR[6,7,8]. Many other features of inflammation including leukostasis, neutrophil and macrophage infiltration, vascular permeability and tissue edema also have been described in animal models and human patients with DR[5,9]. In addition, interlinked molecular pathways, namely increased formation of advanced glycation end-products, oxidative stress, increased expression of VEGF, neurodegeneration have been established the contribution to the inflammatory response[5,9,10]. Moreover, the aforementioned improvement of DR by corticosteroids also supports that inflammation participates the pathophysiology of DR. However, the exact molecular mechanism is not yet fully understood.

Progranulin (PGRN), also known as PC cell-derived growth factor, granulin/epithelin precursor, proepithelin or acrogranin, is a $68-88 \mathrm{kDa}$ glycoprotein which has seven and a half granulin motifs connected by short linker domains[11]. PGRN is synthesized in epithelial cells, immune cells, neurons, adipocytes and so 
forth[11]. The widespread expression of PGRN gene makes it act in many physiological and pathophysiological processes, such as cell growth[12], wound repair[13], tumorigenesis[14], neurodevelopment, neurodegeneration[15], and more recently inflammation[16,17].

It has been shown that PGRN is an adipokine related to obesity-induced insulin resistance(IR), revealing its metabolic function and pro-inflammatory properties[11,16]. Youn et al.[18] revealed that serum PGRN levels were elevated in patients with type 2 diabetes, and correlated with visceral fat accumulation and macrophage infiltration into adipose tissue. Matsubara et al.[16] identified PGRN as a novel pro-inflammatory adipokine by differential proteome analysis of cellular models of IR. They found that PGRN levels were increased in IR state both in vivo and in vitro, and PGRN induced the expression of IL-6, a pro-inflammatory adipokine.

Further study has shown that the role of PGRN in chronic subclinical inflammation contributes to diabetic retinopathy. Xu et al. [19] showed that serum PGRN was significantly increased in the proliferative diabetic retinopathy (PDR) patients compared with the normal controls, simple DM patients, and background diabetic retinopathy (BDR) patients. But later, serum PGRN was found being affected by a decrease in estimated glomerular filtration rate (eGFR) of diabetic kidney disease (DKD) patients with eGFR $<60 \mathrm{ml} / \mathrm{min} / 1.73 \mathrm{~m}^{2}$, but not affected in DKD patients with eGFR $\geq 60 \mathrm{ml} / \mathrm{min} / 1.73 \mathrm{~m}^{2}[20,21,22]$. However, the PDR group in $\mathrm{Xu}$ et al.'s 
research presented reduced eGFR $\left(<60 \mathrm{ml} / \mathrm{min} / 1.73 \mathrm{~m}^{2}\right)$, which may affect their conclusions.

No data to date is available about the association between serum PGRN levels and DR excluding the effect of deteriorating renal function, and the role of PGRN in the pathophysiology of DR is still unknown. Therefore, the primary objective of this study was to investigate the association between serum PGRN levels and DR in patients with type 2 diabetes and eGFR $\geq 60 \mathrm{ml} / \mathrm{min} / 1.73 \mathrm{~m}^{2}$. We also aimed to assess the factors correlated with serum PGRN levels.

\section{Methods}

\subsection{Subjects}

For this cross-sectional study, 262 patients(162 males/100 females) previously or newly diagnosed type 2 diabetes with eGFR $\geq 60 \mathrm{ml} / \mathrm{min} / 1.73 \mathrm{~m}^{2}$ were recruited between January 2015 and August 2016. The patients ranging in age from 18 to 87 years were from the Endocrinology Department of Wuxi People's Hospital Affiliated Nanjing Medical University. The diagnosis of type 2 diabetes was based on the diagnostic criteria of World Health Organization (WHO) in 1999. According to International Clinical Diabetic Retinopathy Disease Severity Scale produced by the International Council of Ophthalmology in 2002, DR was diagnosed and staged as no apparent diabetic retinopathy, mild, moderate, and severe nonproliferative diabetic retinopathy (NPDR), as well as PDR. Patients with fundus diseases of unequal severity in two eyes were staged according to the more severer one[23]. All patients 
were divided into simple diabetes mellitus (SDM) group, NPDR group, and PDR group. The normal control (NC) group included 76 health persons (49 males/27 females) from the examination center of the same hospital.

The following exclusion criteria were applied: age $<18$ years, eGFR $<60$ $\mathrm{ml} / \mathrm{min} / 1.73 \mathrm{~m}^{2}$, pregnancy, past history of malignancy, degeneration disease of the nervous system, acute or chronic inflammation, acute infectious disease, acute surgery or trauma, other endocrine diseases affecting glucose and lipid metabolism, taking thiazolidinedione drugs in 2 weeks, and history of drug abuse.

The study was approved by the local ethical committee. Signed informed consents were obtained from all participants in the study.

\subsection{Methods}

Standardized questionnaires were used to obtain age, sex, diabetes duration, hypertension duration, the history for the present and past illness, and medication. Standardized protocols were used to measure height, body weight, and blood pressure (BP) . Body mass index(BMI) was calculated as weight $/$ height $^{2}\left(\mathrm{~kg} / \mathrm{m}^{2}\right)$.

Overnight fasting blood samples were collected for the determination of hemoglobin A1c (HbA1c), fasting plasma glucose (FPG), fasting insulin (FINS), fasting C-peptide (FCP), blood urea nitrogen (BUN), creatinine (CRE), blood uric acid (BUA), triglyceride (TG), total cholesterol (TC), high-density lipoprotein cholesterol (HDL-C), low-density lipoprotein cholesterol (LDL-C) in a certified laboratory by standard methods. $75 \mathrm{~g}$ oral glucose tolerance tests were performed in 
patients newly diagnosed type 2 diabetes and normal control subjects.

eGFR was estimated by the Chronic Kidney Disease Epidemiology Collaboration Group (CKD-EPI) creatinine equation using an online calculator. The index for homeostasis model assessment of insulin resistance (HOMA-IR) was calculated as follows: FINS(mU/L) $\times \mathrm{FPG}(\mathrm{mmol} / \mathrm{L}) / 22.5$. Triglycerides and glucose (TyG) index was computed using the following formula:

$\ln [\mathrm{FPG}(\mathrm{mmol} / \mathrm{L}) \times 18.015 / 2 \times \mathrm{TG}(\mathrm{mmol} / \mathrm{L}) \times 87.719]$.

Early-morning urine samples were collected for calculating albuminuria/creatinine ratio (ACR).

Serums were separated from the blood samples and frozen at $-80^{\circ} \mathrm{C}$ prior to analysis of PGRN and IL-6. Concentrations of serum PGRN and IL-6 were measured by enzyme linked immunosorbent assay (ELISA) using the commercially available Human ELISA kits (mlbio, CHN) according to the manufacturers' instructions. All samples were diluted to 5 times during testing. The assay sensitivity was $0.08 \mu \mathrm{g} / \mathrm{L}$ and assay range was $0.8-28 \mu \mathrm{g} / \mathrm{L}$ for PGRN, while the assay sensitivity was $0.08 \mathrm{ng} / \mathrm{L}$ and assay range was $0.8-20 \mathrm{ng} / \mathrm{L}$ for IL-6. The specificity of the immunosorbent assay for both PGRN and IL-6 was estimated to be $99.6 \%$.

Fundal examinations were performed on all subjects by a professional ophthalmologist using direct ophthalmoscopy through dilated pupils. Fundus photography was performed on patients with type 2 diabetes, and some confounding cases were finally diagnosed by fundus fluorescein angiography. 


\subsection{Statistical analysis}

SPSS software, version20.0 (IBM, Armonk, NY), was used for all statistical analyses. Overall group differences for continuous parameters were assessed by Kruskal-Wallis test followed by post hoc analysis. Categorical parameters were analyzed using the chi-square test. Univariate correlations were assessed by Spearman rank correlation method. For adjustment for the effects of covariates and identification of independent relationships, multivariate linear stepwise regression analyses were performed. Before multivariate analysis, distribution of continuous variables was tested for normality using One-Sample Kolmogorov-Smirnov test and nonnormally distributed parameters were natural logarithmically transformed. P value $<0.05$ was considered statistically significant in all analyses.

\section{Results}

\subsection{Serum PGRN Levels and Clinical Characteristics of Study Subjects.}

Table 1 summarizes the serum PGRN levels and the other clinical characteristics of the NC, SDM, NPDR, and PDR groups. All continuous variables are given as median (interquartile range). There was no statistical difference between the groups with regard to age and sex. Serum PGRN levels were $25.28 \mu \mathrm{g} / \mathrm{L}$ (interquartile range 9.30) in the total subjects, and there was no significant difference of serum PGRN levels between the four groups $(P>0.05)$. Moreover, there was no significant difference of IL-6 in the four groups either $(P>0.05)$. As compared to NC group, BMI was increased in SDM group, as well as SBP was elevated in SDM and NPDR group 
$(P<0.05$ or $P<0.01)$. The duration of diabetes, FPG, and HbA1c were markedly lower in NC group than in the other three groups $(P<0.01)$, and the duration of diabetes was also significantly lower in SDM group than in NPDR and PDR groups $(P<0.01)$. Some FINS data were not available because of the therapy of insulin, which inducing the missing of the FINS and HOMA-IR in PDR group, but FCP were measured in these patients. There was no statistical difference of FINS in NC, SDM, and NPDR groups $(P>0.05)$, while FCP was significantly lower in PDR group than the other three groups, as well as in NPDR group than in SDM groups $(P<0.05$ or $P<0.01)$. As compared to NC group, HOMA-IR was markedly increased in SDM group, and TyG index was significantly elevated in SDM, NPDR, and PDR groups $(P<0.01)$. There was no statistical difference of BUN, CRE, BUA, eGFR between the four groups $(P>0.05)$, while ACR in NPDR and PDR groups were remarkably higher than NC and SDM groups $(P<0.05$ or $P<0.01)$. TG in SDM group was markedly increased as compared to NC group $(P<0.01)$, while HDL-C in SDM, NPDR, and PDR groups was lower than NC group $(P<0.01)$. There was no significant difference of TC and LDL-C in the four groups $(P>0.05)$.

\subsection{Univariate Correlations between Serum PGRN Levels and Other Metabolic}

\section{Parameters}

Serum PGRN levels in all subjects negatively and significantly correlated with eGFR $(r=-0.144, P<0.05)$, TyG index $(r=-0.127, P<0.05)$, and TG $(r=-0.132, P<0.05)$, while positively and significantly correlated with LDL-C $(r=0.140, P<0.05)$ (Table 2$)$. 
In addition, Serum PGRN was not significantly associated with age, sex, duration of hypertension, duration of diabetes, BMI, SBP, DBP, HBA1C, FPG, FINS, FCP, HOMA-IR, BUN, CRE, BUA, ACR, TC, HDL-C, and IL-6 ( $P>0.05)$ (Table 2).

\subsection{Multiple Stepwise Regression Analysis.}

Multivariate stepwise regression analysis was performed to evaluate the independent factors of serum PGRN with the factors identified in the above univariate analysis including eGFR, TG, and LDL-C as independent variables. The analysis demonstrated that only LDL-C $(\beta=1.030, P<0.05)$ was independently associated with serum PGRN (Table 3). Similar results were obtained when TyG index instead of TG was included in the model (data not shown).

\section{Discussion}

In the present study, we could better investigate the association between serum PGRN levels and DR excluding the influence of reduced eGFR. There was no statistic difference between the SDM, NPDR, and PDR groups with $\mathrm{eGFR} \geq 60 \mathrm{ml} / \mathrm{min} / 1.73 \mathrm{~m}^{2}$.

Previous research regarding PGRN and DR was very few and limited. Xu et al.[19] observed increased serum PGRN levels in PDR patients as compared to SDM and BDR patients, which seemed inconsistent with our results. However, the group of PDR patients in Xu et al.'s research presented reduced eGFR $\left(<60 \mathrm{ml} / \mathrm{min} / 1.73 \mathrm{~m}^{2}\right)$. In a recent study involving 532 patients with stages $1-5$ of chronic kidney disease 
(CKD), Richter et al.[21] demonstrated that serum PGRN levels increased with deteriorating renal function, but with no significantly difference between stage 1 $\left(\mathrm{eGFR} \geq 90 \mathrm{ml} / \mathrm{min} / 1.73 \mathrm{~m}^{2}\right)$ and stage $2\left(60 \leq \mathrm{eGFR}<90 \mathrm{ml} / \mathrm{min} / 1.73 \mathrm{~m}^{2}\right)$ of CKD. Nicoletto et al.[20] also found that PGRN was affected by a decrease in eGFR. Serum PGRN levels was negatively and independently with eGFR in DKD patients with eGFR $<60 \mathrm{ml} / \mathrm{min} / 1.73 \mathrm{~m}^{2}$, but there was no such findings in DKD patients with eGFR $\geq 60 \mathrm{ml} / \mathrm{min} / 1.73 \mathrm{~m} 2$. Taken together, PGRN was a renal function-dependent protein, which may explain the different results between Xu et al.'s and ours.

Even as various research reported previously[20,21], we also observed a negative association between serum PGRN and eGFR. Although, the association was lost in multivariate stepwise regression analysis, which was in accordance with Nicoletto et al.'s research[20]. It seemed that the serum PGRN might be affected by moderate to severe renal insufficiency. However, the mechanism underlying the correlation between PGRN and deteriorating renal function was still unclear. The hypothesis was proposed that renal clearance was an important route of PGRN elimination[20,21,22]. Despite both Richter et al.[21] and Nicoletto et al.[20] did not find a significant correlation between serum and urinary PGRN, it was supposed that PGRN was at least partly degraded during the elimination[21].

In previous research, the association of serum PGRN and DR was regardless of the deteriorating renal function. The development and progression of DR was explained by the increase of serum PGRN, elevated IL-6 and then promoted chronic low-grade inflammation[16]. As we did not observe the significant difference of 
serum PGRN between NC, SDM, NPDR, and PDR groups, we did not find the remarkable difference of serum IL-6 either, and there was no relationship between serum PGRN and IL-6. So the present study did not allow us to identify serum PGRN as a marker for the occurrence and severity of DR. DR is identified not only as a retinal vasculopathy involving the increase of VEGF[24] but also as a retinal neuron injury[25]. Interestingly, the retina is composed of abundant epithelial cells and nerve cells which may secret PGRN[9]. Furthermore, PGRN has been established as a upstream stimulating factor of VEGF concerned in angiogenesis[14], and a neurotrophic and neuroprotective factor involved in central nervous system[15]. However, no data is currently available about the PGRN in ocular samples, i.e., retinal tissue, vitreous and aqueous humor, of DR in humans or animal models.

Previous studies showed that circulating PGRN was increased in patients with type 2 diabetes when compared to subjects without diabetes and there was a positive correlation between circulating PGRN and FPG, HbA1c, as well as HOMA-IR[18,26]. However, these studies did not take eGFR into account. Notably, after controlling for eGFR, the associations between circulating PGRN and FPG, HOMA-IR, as well as other components of the metabolic syndrome including DBP, waist to hip ratio (WHR), waist to height ratio (WHtR), TC, TG, HDL-C, and LDL-C were all lost in the research of Richter et al[21]. Furthermore, both $\mathrm{Xu}$ et al.[19] and Nicoletto et al.[20] did not find a significant difference of serum PGRN between non-diabetic subjects and normoalbuminuric patients without deteriorating eGFR, and there was no relationship between circulating PGRN and FPG, HBA1C, FINS or HOMA-IR. In 
agreement with these studies, no remarkable correlationship was found between serum PGRN and FPG, HbA1c, FINS, HOMA-IR, TyG index in our study.

Other metabolic disorders have also been reported to associate with PGRN[11]. A positive correlation between circulating PGRN and TC, TG indicated PGRN was involved in dyslipidemia[18,17,27]. In this study, we found that LDL-C was positively and independently associated with serum PGRN. LDL-C was acknowledged as a determining risk factor of carotid intima media thickness (CIMT), a useful surrogate marker for atherosclerosis[28]. Yoo et. al.[27] found that serum PGRN was an independent predictor for increased CIMT in subjects without metabolic syndrome after adjusting for other cardiovascular risk factors. Although the exact explanation for the association between PGRN, LDL-C, and CIMT was still unclear, it suggested that PGRN might have an influence on atherosclerosis. Further studies are needed to elucidate the connection.

There were some limitations in our study. Firstly, the sample sizes of NPDR and PDR groups were small. It is quite possible that nonsignificant associations in multivariate analyses would have become statistically remarkable if larger samples were studied. Secondly, our study was a retrospective study, and some data missing might affect the results. Thirdly, the study was a cross-sectional design, and therefore, causality could not be established.

\section{Conclusion}


In conclusion, we demonstrated that serum PGRN levels did not correlate with the occurrence and severity of DR in Chinese patients with type 2 diabetes and eGFR $\geq 60 \mathrm{ml} / \mathrm{min} / 1.73 \mathrm{~m}^{2}$. Furthermore, serum PGRN was positively and independently associated with LDL-C, which suggested that PGRN might be associated with diabetic macrovascular complications. Prospective studies are needed to confirm our observations and to validate the current findings.

\section{Abbreviations}

PGRN: Progranulin; VEGF: Vascular endothelial growth factor; IL-6: Interleukin-6; TNF- $\alpha$ : Tumor necrosis factor- $\alpha$; MCP-1: Monocyte chemoattractant protein-1; eGFR: estimated Glomerular filtration rate; SDM: Simple diabetes mellitus; NC: Normal control; DR: Diabetic retinopathy; NPDR: Nonproliferative diabetic retinopathy; PDR: Proliferative diabetic retinopathy; BDR: Background diabetic retinopathy; DKD: Diabetic kidney disease; CKD: Chronic kidney disease; IR: Insulin resistance; BP: Blood pressure; BMI: Body mass index; HbAlc: Hemoglobin A1c; FPG: Fasting plasma glucose; FINS: Fasting insulin; FCP: Fasting C-peptide; BUN: Blood urea nitrogen; CRE: Creatinine; BUA: Blood uric acid; TG: Triglyceride; TC: Total cholesterol; HDL-C: High-density lipoprotein cholesterol; LDL-C: Low-density lipoprotein cholesterol; ACR: Albuminuria/creatinine ratio; WHR: Waist to hip ratio; WHtR: Waist to height ratio; CIMT: Carotid intima media thickness; CKD-EPI: Chronic Kidney Disease Epidemiology Collaboration Group; HOMA-IR: 
Homeostasis model assessment of insulin resistance; TyG index: Triglycerides and glucose index; ELISA: Enzyme linked immunosorbent assay.

\section{Ethics approval and consent to participate}

The study was approved by the Ethics Committee of the Affiliated Wuxi People's Hospital of Nanjing Medical University (number: RYSX0021), and the acquisition of specimens from all cases was performed with the consent of the patient.

\section{Consent to publish}

Not applicable.

\section{Availability of data and materials}

The datasets used and analysed during the current study are available from the corresponding author on reasonable request.

\section{Competing interests}

The authors declare that they have no competing interests.

\section{Funding}

This work was supported by the National Natural Science Foundation of China (grant number 81500630), and the Wuxi science and technology development Foundation (grant number CSE31N1704). 


\section{Authors' Contributions}

WJW was contributed to the study design. HYC, LX, RFB performed the screening of patients. CJ performed the experiments. HYC, and LJY managed the data. HYC and LX were responsible for the statistical analyses, interpretation of data and drafting the manuscript. WJW reviewed and edited the manuscript. All authors approved the final manuscript.

\section{Acknowledgements}

The authors would like to thank all subjects for their participation in this study and thank medical personnel from Department of Endocrinology, the Affiliated Wuxi People's Hospital of Nanjing Medical University, for their valuable assistance.

\section{References}

1. Zhang X, Saaddine JB, Chou CF, Cotch MF, Cheng YJ, Geiss LS, et al. Prevalence of diabetic retinopathy in the United States, 2005-2008. JAMA 2010; 304: 649-656.

2. Song $\mathrm{P}, \mathrm{Yu}$ J, Chan KY, Theodoratou E, Rudan I. Prevalence, risk factors and burden of diabetic retinopathy in China: a systematic review and meta-analysis. $\mathrm{J}$ Glob Health 2018; 8: 010803.

3. Solomon SD, Chew E, Duh EJ, Sobrin L, Sun JK, VanderBeek BL, et al. Diabetic Retinopathy: A Position Statement by the American Diabetes Association. Diabetes Care 2017; 40: 412-418.

4. Wong TY, Sun J, Kawasaki R, Ruamviboonsuk P, Gupta N, Lansingh VC, et al. Guidelines on Diabetic Eye Care: The International Council of Ophthalmology 
Recommendations for Screening, Follow-up, Referral, and Treatment Based on Resource Settings. Ophthalmology 2018; 125: 1608-1622.

5. Rübsam A, Parikh S, Fort PE. Role of Inflammation in Diabetic Retinopathy. Int J Mol Sci 2018; 19: pii: E942.

6. Feng S, Yu H, Yu Y, Geng Y, Li D, Yang C, et al. Levels of Inflammatory Cytokines IL-1 $\beta$, IL-6, IL-8, IL-17A, and TNF- $\alpha$ in Aqueous Humour of Patients with Diabetic Retinopathy. J Diabetes Res 2018. https://doi.org/10.1155/2018/8546423

7. Koleva-Georgieva DN, Sivkova NP, Terzieva D. Serum inflammatory cytokines IL-1beta, IL-6, TNF-alpha and VEGF have influence on the development of diabetic retinopathy. Folia Med (Plovdiv) 2011; 53: 44-50.

8. Reddy S, Amutha A, Rajalakshmi R, Bhaskaran R, Monickaraj F, Rangasamy S et al. Association of increased levels of MCP-1 and cathepsin-D in young onset type 2 diabetes patients (T2DM-Y) with severity of diabetic retinopathy. J Diabetes Complications 2017; 31: 804-809.

9. Kusuhara S, Fukushima Y, Ogura S, Inoue N, Uemura A. Pathophysiology of Diabetic Retinopathy: The Old and the New. Diabetes Metab J 2018; 42: 364-376.

10. Altmann C, Schmidt MHH. The Role of Microglia in Diabetic Retinopathy: Inflammation, Microvasculature Defects and Neurodegeneration. Int J Mol Sci 2018; 19: pii: E110.

11. Korolczuk A, Bełtowski J. Progranulin, a New Adipokine at the Crossroads of Metabolic Syndrome, Diabetes, Dyslipidemia and Hypertension. Curr Pharm Des 2017; 23: 1533-1539.

12. He Z, Bateman A. Progranulin (granulin-epithelin precursor, PC-cell-derived growth factor, acrogranin) mediates tissue repair and tumorigenesis. J Mol Med (Berl) 2003; 81: 600-612.

13. He Z, Ong $\mathrm{CH}$, Halper J, Bateman A. Progranulin is a mediator of the wound response. Nat Med 2003; 9: 225-229. 
14. Yang D, Wang LL, Dong TT, Shen YH, Guo XS, Liu CY,et al. Progranulin promotes colorectal cancer proliferation and angiogenesis through $\mathrm{T}$ NFR2/Akt and ERK signaling pathways.Am J Cancer Res 2015; 5: 3085-3097.

15. Nguyen AD, Nguyen TA, Martens LH, Mitic LL, Farese RV Jr. Progranulin: at the interface of neurodegenerative and metabolic diseases. Trends Endocrinol Metab 2013; 24: 597-606.

16. Matsubara T, Mita A, Minami K, Hosooka T, Kitazawa S, Takahashi K,Cell Metab. et al. PGRN is a key adipokine mediating high fat diet-induced insulin resistance and obesity through IL-6 in adipose tissue. Cell Metab 2012; 15: $38-50$.

17. Tang W, Lu Y, Tian QY, Zhang Y, Guo FJ, Liu GY,et al. The growth factor progranulin binds to TNF receptors and is therapeutic against inflammatory arthritis in mice. Science 2011; 332: 478-484.

18. Youn BS, Bang SI, Klöting N, Park JW, Lee N, Oh JE, et al. Serum progranulin concentrations may be associated with macrophage infiltration into omental adipose tissue. Diabetes 2009; 58: 627-636.

19. Xu L, Zhou B, Li H, Liu J, Du J, Zang W, et al. Serum Levels of Progranulin Are Closely Associated with Microvascular Complication in Type 2 Diabetes. Dis Markers 2015. https://doi.org/10.1155/2015/357279

20. Nicoletto BB, Krolikowski TC, Crispim D, Canani LH. Serum and Urinary Progranulin in Diabetic Kidney Disease. PLoS One 2016; 11: e0165177.

21. Richter J, Focke D, Ebert T, Kovacs P, Bachmann A, Lössner U, et al. Serum levels of the adipokine progranulin depend on renal function. Diabetes Care 2013; 36: 410-414.

22. Nicoletto BB, Canani LH. The role of progranulin in diabetes and kidney disease. Diabetol Metab Syndr 2015; 7: 117.

23. Wilkinson CP, Ferris FL 3rd, Klein RE, Lee PP, Agardh CD, Davis M, et al. Proposed international clinical diabetic retinopathy and diabetic macular edema disease severity scales. Ophthalmology 2003; 110: 1677-1682. 
24. Simó R, Sundstrom JM, Antonetti DA. Ocular Anti-VEGF therapy for diabetic retinopathy: the role of VEGF in the pathogenesis of diabetic retinopathy. Diabetes Care 2014; 37: 893-899.

25. Stem MS, Gardner TW. Neurodegeneration in the Pathogenesis of Diabetic Retinopathy: Molecular Mechanisms and Therapeutic Implications. Curr Med Chem 2013; 20: 3241-3250.

26. Qu H, Deng H, Hu Z. Plasma progranulin concentrations are increased in patients with type 2 diabetes and obesity and correlated with insulin resistance. Mediators Inflamm 2013. https://doi.org/10.1155/2013/360190

27. Yoo HJ, Hwang SY, Hong HC, Choi HY, Yang SJ, Choi DS, et al. Implication of progranulin and C1q/TNF-related protein-3 (CTRP3) on inflammation and atherosclerosis in subjects with or without metabolic syndrome. PLoS One 2013; 8: e55744.

28. Kent SM, Coyle LC, Flaherty PJ, Markwood TT, Taylor AJ. Marked Low-Density Lipoprotein Cholesterol Reduction below Current National Cholesterol Education Program Targets Provides the Greatest Reduction in Carotid Atherosclerosis. Clin Cardiol 2004; 27: 17-21. 
Table 1 Serum PGRN levels and other Clinical characteristics of the study subjects

\begin{tabular}{|c|c|c|c|c|}
\hline & $\mathrm{NC}$ & SDM & NPDR & PDR \\
\hline$n$ & 76 & 195 & 41 & 26 \\
\hline PGRN $(\mu \mathrm{g} / \mathrm{L})$ & $26.37(8.28)$ & $24.50(8.68)$ & $27.55(11.76)$ & $23.13(13.26)$ \\
\hline Age (years) & $55.0(17.8)$ & $58.0(17.0)$ & $57.0(14.0)$ & $60.5(11.8)$ \\
\hline Sex (male/female) & $49 / 27$ & $127 / 68$ & $23 / 18$ & $12 / 14$ \\
\hline Duration of hypertension(years) & $0(0)$ & $1.0(10.0)^{*}$ & $2.0(11.0)$ & $0(4.8)$ \\
\hline Duration of diabetes (years) & $0(0)$ & $2.0(9.0)^{* *}$ & $12.0(7.5)^{* * \# \#}$ & 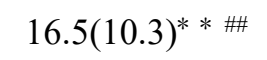 \\
\hline BMI $\left(\mathrm{kg} / \mathrm{m}^{2}\right)$ & $23.12(3.81)$ & $24.42(3.57)^{* *}$ & $23.34(3.15)$ & $23.19(3.21)$ \\
\hline $\mathrm{SBP}(\mathrm{mmHg})$ & $120(20)$ & $130(17)^{* *}$ & $130(20)^{*}$ & $130(13)$ \\
\hline DBP (mmHg) & $76(13)$ & $80(0)$ & $80(8)$ & $80(10)$ \\
\hline HbA1c $(\%)$ & $5.4(0.8)$ & $8.2(3.1)^{* *}$ & $8.7(3.2)^{* *}$ & $9.2(2.8)^{* *}$ \\
\hline FPG (mmol/L) & $5.29(0.62)$ & $8.10(4.77)^{* *}$ & $7.77(5.92)^{* *}$ & $8.92(4.07)^{* *}$ \\
\hline FINS (mU/L) & $7.39(5.52)$ & $8.64(5.97)$ & $7.10(7.54)$ & - \\
\hline FCP $(n g / m l)$ & $2.38(0.79)$ & $2.14(1.29)$ & $1.86(1.39)^{\#}$ & $0.95(0.75)^{* * \# \# \triangle}$ \\
\hline HOMA-IR & $1.8(1.3)$ & $3.5(2.7)^{* *}$ & $1.9(2.0)$ & - \\
\hline TyG index & $8.5(0.7)$ & $9.3(1.0)^{* *}$ & $9.3(1.5)^{* *}$ & $9.2(1.4)^{* *}$ \\
\hline BUN（mmol/1） & $4.3(1.7)$ & $4.5(1.8)$ & $4.9(1.9)$ & $4.8(1.3)$ \\
\hline $\mathrm{CRE}(\mu \mathrm{mol} / \mathrm{L})$ & $75.6(21.0)$ & $67.4(22.1)$ & $64.1(26.8)$ & $67.6(19.2)$ \\
\hline $\mathrm{BUA}(\mu \mathrm{mol} / \mathrm{L})$ & $322.2(114.4)$ & $282.0(93.3)$ & 275.1(139.6) & $250.8(123.9)$ \\
\hline eGFR (ml/min $\left./ 1.73 \mathrm{~m}^{2}\right)$ & $102.1(22.0)$ & $99.7(22.3)$ & $99.0(18.7)$ & $102.7 .0(17.9)$ \\
\hline ACR $(\mathrm{mg} / \mathrm{g})$ & $5.09(4.25)$ & $12.26(30.68)$ & $30.59(93.64)^{* * \#}$ & $27.47(85.94)^{* * \#}$ \\
\hline TG (mmol/L) & $1.14(0.84)$ & $1.74(1.45)^{* *}$ & $1.48(2.15)$ & $1.25(1.43)$ \\
\hline $\mathrm{TC}(\mathrm{mmol} / \mathrm{L})$ & $4.70(1.04)$ & $4.48(1.26)$ & $4.75(1.45)$ & $4.53(1.19)$ \\
\hline HDL-c(mmol/L) & $1.22(0.46)$ & $0.96(0.32)^{* *}$ & $0.99(0.28)^{* *}$ & $0.90(0.35)^{* *}$ \\
\hline LDL-C(mmol/L) & $2.69(1.00)$ & $2.61(1.02)$ & $2.69(1.10)$ & $2.58(1.04)$ \\
\hline IL-6 (ng/L) & $19.75(6.65)$ & $20.37(6.54)$ & $19.24(11.37)$ & $21.77(9.64)$ \\
\hline
\end{tabular}

Data are presented as median (interquartile range). Continuous parameters were analyzed by 
Kruskal-Wallis test followed by post hoc analysis. Categorical parameters were analyzed using the $\chi 2$ test. PGRN: progranulin, BMI:body mass index, SBP: systolic blood pressure, DBP: diastolic blood pressure, HbA1c: hemoglobin A1c, FPG: fasting plasma glucose, FINS: fasting serum insulin, FCP: fasting C-peptide, HOMA-IR: Homeostasis Model Assessment for Insulin Resistance, TyG: triglycerides and glucose index, BUN: blood urea nitrogen, CRE: creatinine, BUA: blood uric acid, eGFR: estimated glomerular filtration rate, ACR: albuminuria/creatinine ratio, TG: triglyceride, TC: total cholesterol, HDL-C: high-density lipoprotein cholesterol, LDL-C: low-density lipoprotein cholesterol, IL-6: interleukin-6.

NC: normal control, SDM: simple diabetes mellitus, NPDR: nonproliferative diabetic retinopathy, PDR: proliferative diabetic retinopathy.

Compared with NC, ${ }^{*} P<0.05,{ }^{* *} P<0.01$, Compared with SDM, ${ }^{\#} P<0.05,{ }^{\#} P<0.01$, Compared with BDR, ${ }^{\triangle} P<0.05,{ }^{\triangle} P<0.01$.

Table 2 Univariate correlations with serum PGRN in the study subjects

\begin{tabular}{lccc}
\hline & $n$ & $r$ & $P$ \\
\hline Age & 338 & 0.078 & 0.154 \\
Sex & 338 & 0.049 & 0.371 \\
Duration of hypertension & 226 & -0.068 & 0.312 \\
Duration of diabetes & 338 & 0.047 & 0.392 \\
BMI & 273 & -0.049 & 0.418 \\
SBP & 280 & -0.036 & 0.549 \\
DBP & 280 & -0.011 & 0.853 \\
HbA1c & 275 & -0.047 & 0.434 \\
FPG & 333 & -0.030 & 0.586 \\
FINS & 149 & 0.002 & 0.979 \\
FCP & 227 & -0.036 & 0.587 \\
HOMA-IR & 147 & -0.050 & 0.545 \\
TyG index & 322 & -0.127 & $0.023^{*}$
\end{tabular}




\begin{tabular}{llll} 
BUN & 252 & 0.051 & 0.423 \\
CRE & 317 & 0.017 & 0.763 \\
BUA & 245 & -0.027 & 0.673 \\
eGFR & 317 & -0.144 & $0.010^{*}$ \\
ACR & 187 & -0.050 & 0.497 \\
TG & 325 & -0.132 & $0.017^{*}$ \\
TC & 326 & 0.055 & 0.324 \\
HDL-C & 324 & 0.079 & 0.157 \\
LDL-C & 325 & 0.140 & $0.012^{*}$ \\
IL-6 & 338 & -0.065 & 0.230 \\
\hline
\end{tabular}

n,subjects included in each correlation analysis. ${ }^{*} P<0.05$ as assessed by Spearman correlation method.

Table 3 Multiple stepwise regression analysis showing variables independently associated with

\begin{tabular}{ccccccc}
\multicolumn{7}{c}{ serum levels of PGRN. } \\
\hline \multirow{2}{*}{$\begin{array}{c}\text { Independent } \\
\text { variable }\end{array}$} & \multicolumn{2}{c}{ Unstandardized } & Standardized & & \\
& variable enter the & coefficients & coefficients & $t$ & $P$ \\
& model & $B$ & Std. error & Beta & & \\
\hline \multirow{2}{*}{ PGRN } & (Constant) & 22.976 & 1.281 & & 17.930 & 0.000 \\
& LDL-C & 1.030 & 0.459 & 0.125 & 2.241 & 0.026 \\
\hline
\end{tabular}

